\title{
Le renouveau ... et la continuité.
}

J.C. Czyba m'a confié qu'il redoutait que l'éditorial du numéro de Septembre 2000 ait un arrière-goût "nécrologique". Me voici interdit d'hommages et d'éloges. Je me dois néanmoins d'exprimer ici les plus sincères remerciements des Andrologues de Langue Française au Rédacteur en Chef de leur revue depuis 1993.

Par-delà les remerciements, une pointe d'inquiétude risque de s'insinuer dans le cœur des andrologues de langue française, ANDROLOGIE supportera-t-elle le départ de J.C. Czyba ? J'ose tenter de rassurer le lecteur. Parmi les nombreuses raisons que l'on a de remercier J.C. Czyba, la principale est qu'il a su mettre en place l'avenir, pour qu'il puisse ... changer de vie... sans que tout ne soit à reconstruire derrière lui. S'il a déclaré depuis quelques années, qu'au 1er Septembre 2000, il cessait toute activité professionnelle, y compris la fonction de rédacteur en chef d'Andrologie, cela n'a jamais voulu dire "après moi le déluge", tout au contraire, pour ANDROLOGIE comme pour ses autres activités, les successeurs se sentent prêts. Certes on sait bien qu'il nous faudra déployer maints efforts pour maintenir la qualité de ce qui a été mis en place, certes le charisme va manquer, mais on se sent prêt au renouveau ... et la continuité, sur les bases mises en place avec J.C. Czyba.

Je remercie par avance les membres du Conseil d'administration de la SALF, du comité de rédaction de la revue, l'équipe éditoriale et les futurs auteurs de leur aide pour assurer la continuité d'ANDROLOGIE.

\section{H. Lejeune}

